\title{
Resolución espontánea de quiste aracnoideo intracraneal asintomático
}

\author{
M. Gelabert-González*; R. Serramito-García y A. García-Allut
}

Instituto Universitario de Ciencias Neurológicas "Pedro Barrié de la Maza"*. Departamento de Cirugía. Facultad de Medicina. Universidad de Santiago de Compostela.

\section{Resumen}

Los quistes aracnoideos son lesiones benignas, de origen congénito y de localización extraparenquimatosa, que con frecuencia se diagnostican de forma incidental en niños y adultos. Su etiología e historia natural no están perfectamente establecidas en la actualidad. La introducción de la tomografía computarizada y resonancia magnética han condicionado un incremento en el número de casos diagnosticados, muchos de ellos de forma incidental. En la literatura existen algunas referencias sobre la desaparición espontánea de quistes aracnoideos intracraneales.

Presentamos el caso clínico de un quiste aracnoideo silviano que se redujo progresivamente hasta su resolución a lo largo de un período de 13 años. Revisamos los casos publicados previamente y analizamos los posibles mecanismos causante de su resolución espontánea.

PALABRAS CLAVE: Fosa craneal media. Quiste aracnoideo. Resonancia magnética. Resolución espontánea.

Spontaneous resolution of an asymptomatic intracranial arachnoid cyst

\section{Summary}

Arachnoid cysts are commonly considered to be benign, congenial, extraparenchymatous anomalies. Small cyst are common incidental findings in children and adults. The aetiology and natural history of arachnoid cysts are not fully understood. In most cases, the presence of the cysts is detected on CT-scans or MRI performed for other reasons. In the literature, there have been few documented cases of arachnoid cysts with spontaneous regression. We reports the case of a silvian arachnoid cyst, which disappeared spontaneously during the 13-year-follow-up period. We review the cases previously reported and the mechanisms

Recibido: 20-12-07. Aceptado: 12-02-08 underlying the resolution of the arachnoid cysts are discussed.

KEY WORDS: Arachnoid cysts. Magnetic resonance imaging. Middle fossa. Spontaneous disappearance.

\section{Introducción}

Los quistes aracnoideos (QA) constituyen alrededor del $1 \%$ de los procesos intracraneales ocupantes de espacio en la edad pediátrica. Menos de la mitad de los casos presentan sintomatología que indique la necesidad de un tratamiento quirúrgico bien mediante un proceso derivativo o un abordaje directo con craneotomía ${ }^{7}$.

En un número considerable de pacientes, el diagnóstico del QA es incidental al practicar un estudio de imagen por otro motivo y en estos casos, en los que no existe sintomatología atribuida al QA, debe optarse por una actitud conservadora y controlar su evolución.

Se han descrito casos de crecimiento del quiste ${ }^{10}$, de ruptura espontánea o traumática del mismo e incluso su remisión natural, llegando a su desaparición completa en las pruebas de imagen ${ }^{6,7}$.

Presentamos el caso de un joven de 18 años de edad, con un QA silviano que se diagnosticó tras un traumatismo craneal y en el que no se indicó el tratamiento quirúrgico. Se realizó seguimiento clínico y radiológico evidenciando 13 años más tarde la desaparición del quiste. Se realiza una revisión de la literatura y se analizan los posibles mecanismos patogénicos de este fenómeno.

\section{Caso clínico}

Varón de 18 años que sufre accidente de circulación (ocupante de vehículo), en el año 1993, golpeándose en la cabeza y cara. Tras su ingreso en el hospital, estaba consciente y no se objetivaban déficits neurológicos. Presentó una fractura de maxilar que fue tratada quirúrgicamente.

Se realizó una TC craneal que mostraba un quiste aracnoideo localizado en la cisura silviana izquierda (tipo II de Galassi). El volumen estimado del quiste fue de $55 \mathrm{cc}$ 


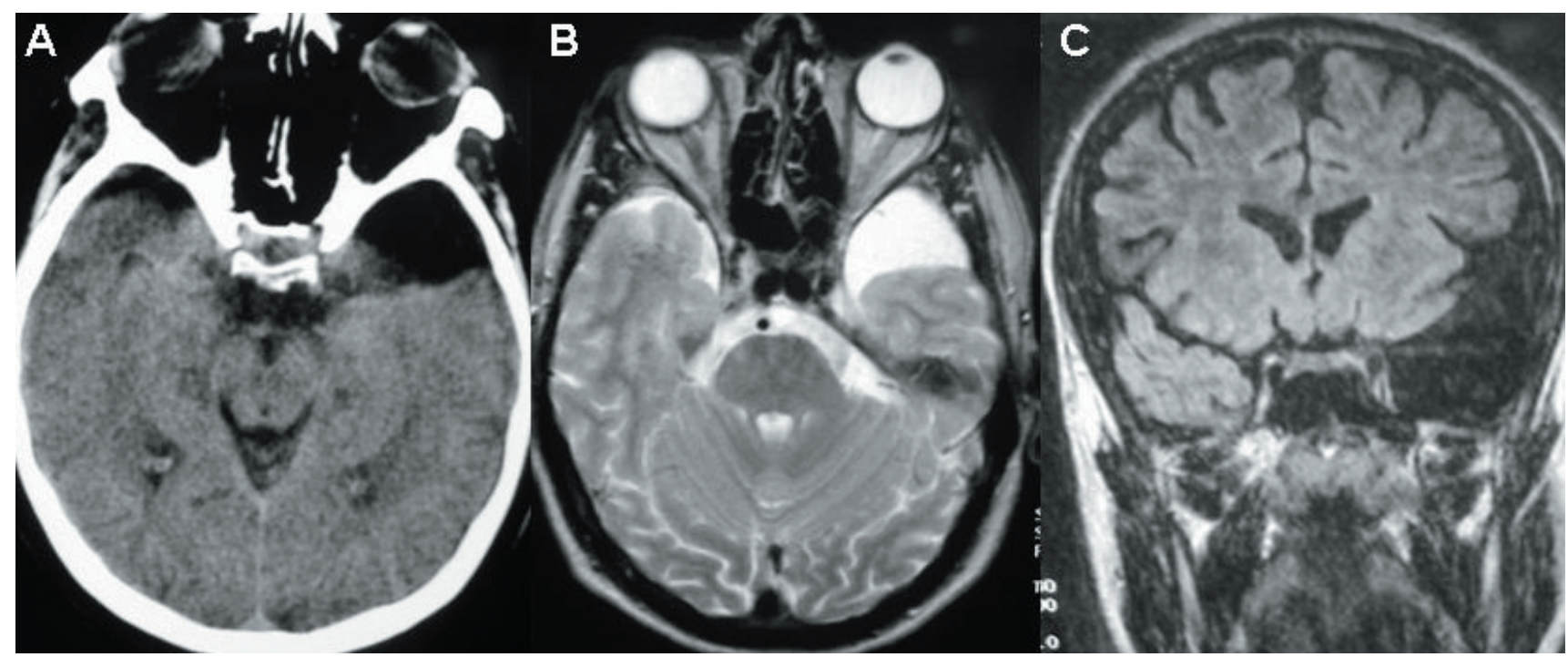

Figura 1. (A) TC realizado en 1993 mostrando el quiste aracnoideo Silviano. (B-C) RM practicada en el año 2003, se observa la persistencia del quiste.

(Figura 1, A). No se indicó ninguna actuación terapéutica sobre el QA aconsejándose controles periódicos.

En los años siguientes presentó consumo de heroína, cocaína y cannabis fumada e importante etilismo. Así mismo, tuvo alteraciones del comportamiento siendo diagnosticado de trastorno esquizofreniforme siguiendo controles periódicos en psiquiatría. En los años 2003 y 2005 se realizan TC y RM craneales observándose la persistencia del quiste aracnoideo silviano izquierdo (Figura 1, B-C).

En marzo de 2006, a los 31 años de edad, reingresó por un nuevo brote esquizofrénico y se repite la RM, que evidenció una reducción muy notable del quiste aracnoideo, representando únicamente un pequeña lesión en la punta del lóbulo temporal (Figura 2). El interrogatorio exhaus- tivo del paciente no evidenció que hubiese sufrido nuevos traumatismos, episodios mantenidos de cefalea o infección meníngea. Clínicamente no se evidenciaron cambios notables en el trastorno psiquiátrico que presentaba el paciente.

\section{Discusión}

Los QA son colecciones extracerebrales de carácter benigno formados por líquido cefalorraquídeo, que están rodeados por una membrana aracnoidea indistinguible histológicamente de la aracnoides sana ${ }^{7}$. Aunque se pueden presentar a cualquier edad, son característicos de la infancia, etapa en la que representan el $1 \%$ de todos los procesos intracraneales ocupantes de espacio. La evolución de los

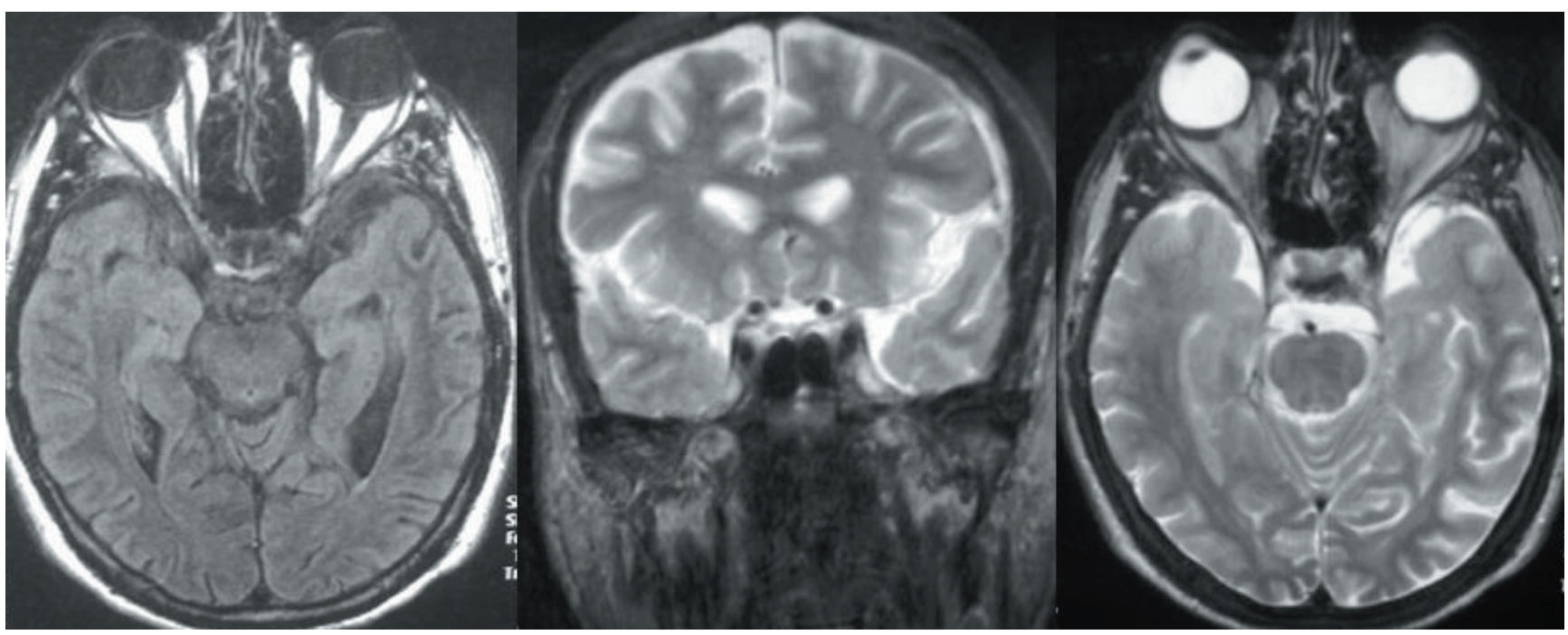

Figura 2. RM realizada en marzo de 2006 mostrando una resolución prácticamente completa del quiste aracnoideo. 

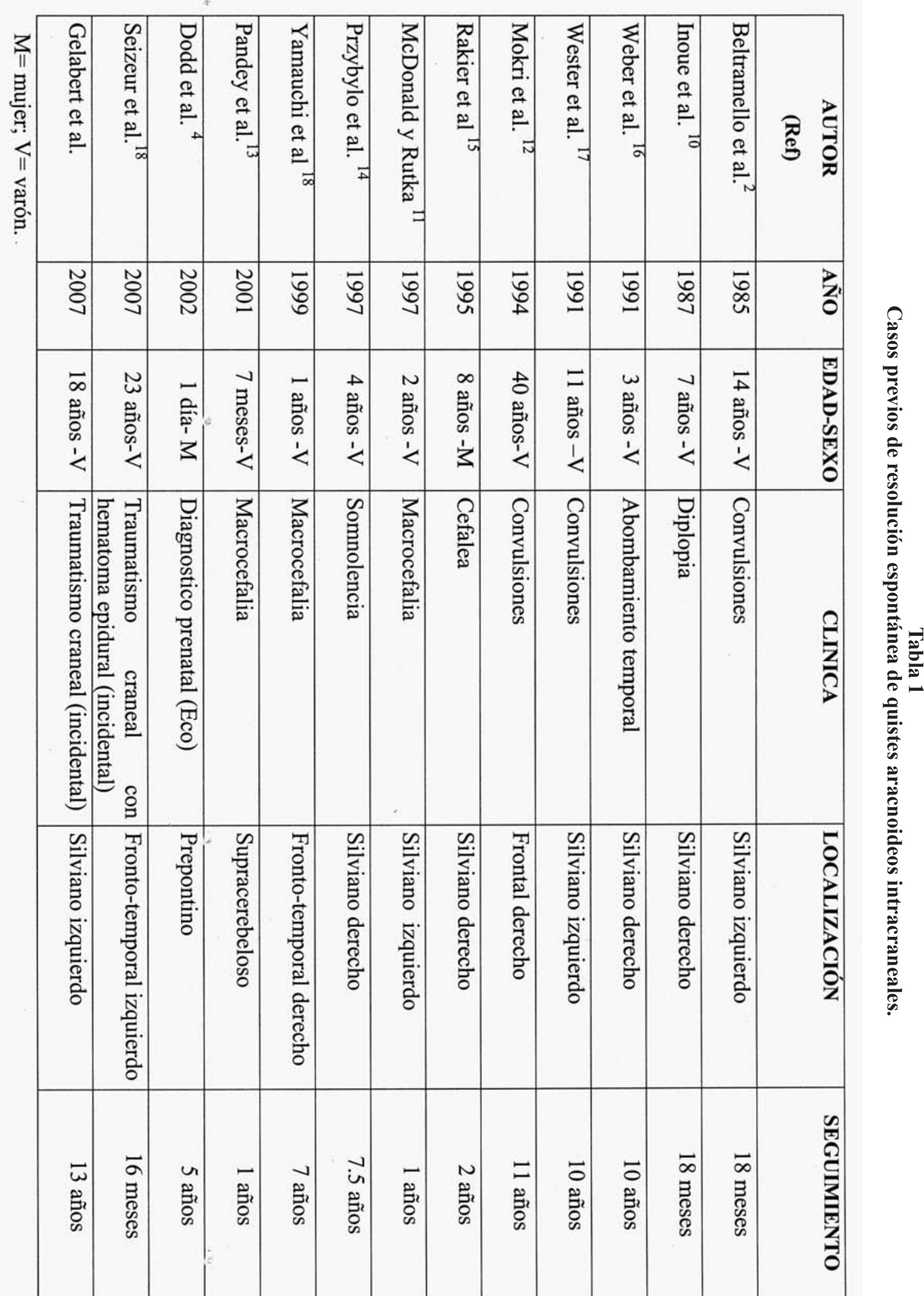
quistes aracnoideos es impredecible, pudiendo permanecer asintomáticos durante largos períodos de tiempo, crecer de forma lentamente progresiva, o desaparecer tras un traumatismo, infección intracraneal o menos frecuentemente de forma espontánea ${ }^{1,3,10}$. Esta última opción es excepcional, existiendo únicamente 12 casos recogidos en la literatura $^{2,4,9-18}$ (Tabla 1).

La edad media en el momento del diagnostico es de 9 años (rango 1 día - 40 años), siendo 10 varones y 2 mujeres. Excepto un caso, en todos los pacientes publicados, existía algún síntoma que indicase la necesidad de un estudio de neuroimagen; sin embargo, nuestro paciente estaba asintomático y fue un traumatismo facial el que indico la necesidad del TC.

Ocho de los casos publicados estaban situados en la región silviana ${ }^{2,9,11,13-16,18}$ donde en general, asientan el 50\% de todos los QA; dos se localizaban en otros puntos de la convexidad $^{11,17}$ y otros dos estaban alojados en la fosa craneal posterior ${ }^{4,12}$. El proceso de resolución de los quistes fue gradual en 8 casos y en un período de tiempo que osciló entre 6 meses y 10 años $2,4,9,11,13-15,18$, aunque en ocasiones no existen estudios de imagen intermedios entre el diagnóstico y su resolución ${ }^{10,12,16,17}$.

Los mecanismos para explicar la desaparición de estos quistes, en ausencia de traumatismos, hemorragias o procesos infecciosos son controvertidos. Para algunos autores, es posible que el paciente haya sufrido un traumatismo craneal que puede no ser recogido, sobre todo en los casos infantiles, y que este trauma ocasione la ruptura del quiste y su evacuación hacia el espacio subaracnoideo. En la misma línea Yamauchi et $\mathrm{al}^{17}$ piensan que es posible que los niños al llorar o con repetidas maniobras de Valsalva aumenten la presión intraquística ocasionando la ruptura espontánea de la membrana aracnoidea y su comunicación con los espacios subaracnoideo o subdural (creación de una fenestración espontánea del quiste), mecanismo fácilmente explicable en los quistes localizados en la cisura Silviana (8 casos).

En nuestro caso, aunque no hay una evidencia clara de que el paciente sufriera un traumatismo craneal (al menos de cierta envergadura), dados los antecedentes psiquiátricos del paciente, no se puede descartar de forma rotunda algún tipo de evento traumático que el paciente no relatase.

\section{Bibliografía}

1. Becker, T., Wagner, M., Hofmann, E., Warmuth-Metz, M., Nadjmi, M.: Do arachnoid cyst grow? A retrospective volumetric study. Neuroradiology 1991; 33: 341-345.

2. Beltramello, A., Mazza, C.: Spontaneous disappearance of a large middle fossa arachnoid cyst. Surg Neurol 1985; 24: 181-183.

3. Coffey, R.J., Lunsford, L.D.: Supracallosal interhemispheric arachnoid cyst: Resolution after intracystic haemorrhage and infection. Surg Neurol 1988; 29: 153-158.
4. Dodd, R.L., Barnes, P.D., Huhn, S.L.: Spontaneous resolution of a prepontine arachnoid cyst. Case report and review of the literature. Pediatr Neurosurg 2002; 37: 152-157.

5. Galassi, E., Piazza, G., Gaist, G., Frank, F.: Arachnoid cysts of the middle cranial fossa. A clinical and radiological study of 25 cases treated surgically. Surg Neurol 1980; 14: 211-219.

6. Gelabert-González, M., Fernández-Villa, J., CutrínPrieto, J., García-Allut, A., Martínez-Rumbo, R.: Arachnoid cyst rupture with subdural hygroma. Report of three cases and literature review. Childs Nerv Syst 2002; 18: 609-613.

7. Gelabert-González, M.: Quistes aracnoideos. Rev Neurol 2004; 39: 1161-1166.

8. Hayakawa, H., Ohkuma, A., Hattori, S., Niikawa, S., Kobayashi, H.: Primary intracranial arachnoid cyst in the elderly: a survey on 39 cases. Acta Neurochir (Wien) 1991; 113: 42-47.

9. Inoue, T., Matushima, T., Tashima, S., Fukui, M., Hasuo, K.: Spontaneous dissappearance of a middle fossa arachnoid cyst associated with subdural hematoma. Surg Neurol 1987; 28: 447-450.

10. McDonald, P.J., Rutka, J.T.: Middle cranial fossa arachnoid cysts that come and go. Report of two cases and review of the literature. Pediatr Neurosurg 1997; 26: 48-52.

11. Mokri, B., Houser, W.O., Dinapoli, R.P.: Spontaneous resolution of arachnoid cyst. J Neuroimaging 1994; 4: 165-168.

12. Pandey, P., Tripathi, M., Chandra, P.S., Singh, V.P., Metha, V.S.: Spontaneous decompression of a posterior fossa arachnoid cyst: a case report. Pediatr Neurosurg 2001; 35: 162-163.

13. Przybylo, H.J., Radkowski, M.A., Przybylo, J., McLone, D.: Spontaneous resolution of an asymptomatic arachnoid cyst. Pediatr Neurosurg 1997; 26: 312-314.

14. Rakier, A., Feinsod, M.: Gradual resolution of an arachnoid cyst after spontaneous rupture into the subdural space. J Neurosurg 1995; 83: 1085-1086.

15. Weber, R., Voit, T., Lumenta, C., Lenard, H.G.: Spontaneous regression of a temporal arachnoid cyst. Childs Nerv Syst 1991; 7: 414-415.

16. Wester, K., Gilhus, E.N., Hugdahl, K., Lasen, L.: Spontaneous disappearance of an arachnoid cyst in the middle fossa. Neurology 1991; 41: 1524-1526.

17. Yamauchi, T., Saeki, N., Yamaura, A.: Spontaneous disappearance of temporo-frontal arachnoid cyst in a child. Acta Neurochir (Wien) 1999; 141: 537-540.

18. Seizeur, R., Forlodou, P., Coustans, M., Dam-Hieu, P.: Spontaneous resolution of arachnoid cyst: review and features of an unusual case. Acta Neurochir (Wien) 2007; 149: 75-78.

Gelabert-González,M.; Serramito-García, R.; García-Allut, A.: Resolución espontánea de quiste aracnoideo intracraneal asintomático. Neurocirugía 2008; 19: 361-364.

Correspondencia postal: Miguel Gelabert González. Instituto de Ciencias Neurológicas. Facultad de Medicina. San Francisco 1. 15705 Santiago de Compostela 